\title{
CONSENSUS FOR MULTIPLE UNMANNED SURFACE VEHICLE (MUSV) SYSTEMS WITH MARKOV SWITCHING TOPOLOGIES
}

\author{
Liyuan Wang ${ }^{1,2}$ \\ Wei Yue ${ }^{3}$ \\ Rubo Zhang ${ }^{1,2}$ \\ ${ }^{1}$ Key Laboratory of Intelligent Perception and Advanced Control State Ethnic Affairs Commission, Dalian, China \\ ${ }^{2}$ College of Mechanical and Electronic Engineering, Dalian Minzu University, Dalian, China \\ ${ }^{3}$ Marine Electrical Engineering College, Dalian Maritime University, Dalian, China
}

\begin{abstract}
This paper is concerned with sampled-data leader following consensus of multiple unmanned surface vehicle (MUSV) systems with random switching network topologies and wave-induced disturbance. By modelling the switching of network topologies with the use of a Markov process and considering the effect of wave-induced disturbance, a new sampleddata consensus control protocol is proposed. By employing an appropriate Lyapunov-Krosovskii function method and the weak infinitesimal operation, a novel stability criterion is derived, which ensures that the MUSV system can reach robustly leader-following consensus with $H_{\infty}$ performance satisfied. Based on this criterion, the Markov dependent switching consensus controller gains are obtained by solving a set of linear matrix inequalities. Finally, an illustrative example is given to verify the effectiveness of the proposed control scheme for MUSV systems.
\end{abstract}

Keywords: Consensus, unmanned surface vehicle (USV), Markov process, wave-induced disturbance

\section{INTRODUCTION}

During the past few years, formation control of multi-agent systems attracted great attention in system and control areas. This is partially due to the fact that there is an increasing need for utilizing multiple agents to perform difficult tasks, where it contributes to increasing efficiency, reducing system cost and providing redundancy against individual failure. To achieve the desired formation, several methods have been proposed but the leader-follower strategy seems to be much preferred in practice due to its simplicity and scalability [1].

As a special case of leader-following formation control, leader-following consensus control can enable vehicle's states or outputs to approach consistency. In the past few years, leader-following consensus control has attracted intensive attention for various forms of multi-agent systems [2-4]. However, the controlled plants are limited to general linear systems, unmanned aerial vehicles and robots. Few results are available in the literature discussing the consensus of MUSV systems. In particular, the vehicles in the marine environment are exposed to waved-induced disturbance, this way the problem becomes even more difficult. Hence, it is important to establish a dynamic USV model and the leader-following consensus control method for MUSV systems by taking the wave-induced disturbance into consideration. This gives rise to the first motivation of the current work.

With the rapid development of communication technologies, network-based multi-agent systems have been attractive, in which agents communicate with each other through a communication network [5]. However, due to the communication constraints, such as link failures, packet dropouts, external disturbances, channel fading, task execution alteration, etc, the connectivity of network topology might be randomly switching among multiple topologies in a deterministic manner. Thus, recent attention has been paid to consensus control of networked multi-agent systems in a stochastic framework, where the switching of the network topologies is described by a Bernoulli or Markov process. To mention a few, the consensus problem for a class of secondorder multi-agent systems with Markovian characterizations is considered in [6], where the stochastic switching topology and the 
random communication delay are dominated by two mutually independent Markov chains. A more general case with highorder nonlinear multi-agent systems is investigated in [7] and [8]. Different from [6-8], the consensus problem for heterogeneous linear time-invariant (LTI) networked multi-agent systems with switching topology and time-varying delays is discussed in [9], where a novel two-stage distributed consensus protocol is proposed. To reduce unnecessary communication, an eventtriggered leader-following consensus problem for a multi-agent system with semi-Markov switching topologies is investigated in [10]. However, all the above consensus control methods do not conform to the actual engineering situation in the marine environment with randomly switching topologies and the robust consensus problem of MUSV systems has not been solved so far. This is the second motivation of the current work.

In light of the aforementioned statements, this paper aims to investigate sampled-data leader-following consensus of MUSV systems with randomly switching network topologies and waveinduced disturbance. Each USV is assumed to have access to its own states (including sway velocity, yaw velocity, heading angle, roll velocity and roll angle) and those of its neighbours at each sampling time. All sampled-data are transmitted through a communication network whose topology is steered by a Markov process. For such systems, we first present a sampleddata leader-following consensus protocol with "sampled" network topologies at each sampling time. Then, by using an appropriate Lyapunov-Krosovskii approach and the weak infinitesimal operation, sufficient condition is derived, which guarantees that the MUSV systems can reach robustly stochastic leader-following consensus with $H_{\infty}$ performance satisfied. Moreover, the "topology-dependent" sampled-data consensus controller gains are obtained based on the condition. Finally, a numerical example is given to illustrate the effectiveness of the results derived in this paper.

Notation: The superscript $T$ represents matrix transposition. $\mathrm{N}$ is the set of natural numbers. $\mathbb{R}^{n}$ denotes the $n$-dimensional Euclidean space. $I_{n} \in \mathrm{R}^{n}$ is an identity matrix. $\operatorname{diag}\left\{a_{i}\right\}$ is a diagonal matrix with diagonal entries $a_{i} . P>0$ means that matrix $P$ is symmetric positive definite. The symbol $*$ denotes the symmetric terms in a symmetric matrix.

\section{PROBLEM FORMULATION}

In this section, we first introduce some basic notions of graph theory. Then the dynamics of USV is presented. Finally, we state a leader-following consensus problem of MUSV systems with Markov switching topologies.

\section{GRAPH THEORY}

Let $G=\{\Delta, S, W\}$ denote a directed weighted graph of $N$ - order, where $\Delta=\left\{v_{1}, v_{2}, \ldots, v_{N}\right\}$ and $S \subseteq \Delta \times \Delta$ are the set of nodes and edges, respectively, $W=\left[w_{i j}\right] \in \mathrm{R}^{n}$ represents the weighted adjacency matrix with $w_{i i}=0$ for any $i$. An edge defined as $s_{i j}=\left(v_{i}, v_{j}\right)$ implies that node $v_{i}$ can receive information from node $v_{i}$. Node $v_{j}$ is considered as a neighbour of node $v_{i}$ if $s_{i j} \in S$. The degree matrix of sub-graph $G$ is denoted by $\bar{W}=\operatorname{diag}\left\{w_{1}, w_{2}, \ldots, w_{N}\right\}$, where the diagonal element is represented as $w_{i}=\sum^{N}{ }_{j=1} w_{i j}$ (which is also called the in-degree of node). Correspondingly, the Laplace matrix of the directed graph $G$ is defined as $L=\bar{W}-W$. A path is a sequence of connected edges in a graph. If there exists a path between node $v_{i}$ and node $v_{j}$, node $v_{i}$ in the graph is said to be reachable from another node $v_{j}$. The union graph of a collection of directed graph $G_{r}(r=1,2, \ldots, s)$, which is denoted by $\bigcup_{r=1}^{s} G_{r}$, is a directed graph with node set $\Delta$ and the edge set equal to the union of the edge sets of all of the graphs $G_{r}$ in the collection.

\section{USV DYNAMICS}

The motion of a ship with six degrees of freedom includes sway, yaw, roll, surge, heave, and pitch $[11,12]$. The main concern of this paper is the motion in sway, yaw, and roll. The influence of surge, heave, and pitch is treated as disturbance. Applying Newton's laws in a space-fixed coordinate system, one can have the equations for sway, yaw and roll as follows :

$$
\left\{\begin{array}{l}
m_{y_{a}} \frac{d^{2} y_{a}}{d t^{2}}=F_{y_{a}}, \text { sway } \\
I_{z z_{a}} \frac{d^{2} h_{a}}{d t^{2}}=N_{a}, \text { yaw } \\
I_{x x_{a}} \frac{d^{2} f_{a}}{d t^{2}}=K_{a}, \text { roll }
\end{array},\right.
$$

where $x_{a}, y_{a}$, and $z_{a}$ denote the longitudinal xis, transverse axis, and normal axis, respectively; $m_{y_{a}}$ and $F_{y_{a}}$ denote the effective mass and the force of the ship in the $y_{a}$ - direction, respectively; $I z z_{a}$ and $I x x_{a}$ denote moments of inertia with respect to the $z_{a}$ and $x_{a}$ axes, respectively; $h_{a}$ and $f_{a}$ denote the heading angle and the roll angle, respectively; $N_{a}$ and $K_{a}$ denote moments with respect to the $z_{a}$ and $x_{a}$ axes, respectively.

By translating the equation system (1) to the motion coordinate system utilizing Taylor series expansions, Laplace transformation, and a model simplification, one can obtain the following state-space model [13]

$$
\dot{x}(t)=A x(t)+B u(t)+C w(t),
$$

where $x(t)=\left[\begin{array}{lllll}v(t) & r(t) & \psi(t) & p(t) & \phi(t)\end{array}\right]^{T} \in \mathrm{R}^{n}$ with $v(t), r(t), \psi(t)$, $p(t)$ and $\phi(t)$ denoting the sway velocity caused by the rudder motion alone, yaw velocity, heading angle, roll velocity, and roll angle, respectively; $u(t)$ represents the rudder angle; $w(t)=\left[w_{\psi}(t) w_{\phi}(t)\right]^{T}$ denotes the wave-induced disturbance, and $w(t) \in L_{2}\left[t_{0}, \infty\right)$ with $t_{0}$ denoting the initial instant; $x_{0} \in \mathrm{R}^{n}$ denotes the initial condition; $A, B$, and $C$ are given by:

$$
A=\left[\begin{array}{ccccc}
-1 / T_{v} & 0 & 0 & 0 & 0 \\
K_{v r} / T_{r} & -1 / T_{r} & 0 & 0 & 0 \\
0 & 1 & 0 & 0 & 0 \\
w_{n}^{2} K_{v p} & 0 & 0 & -2 \varsigma w_{n} & -w_{n}^{2} \\
0 & 0 & 0 & 1 & 0
\end{array}\right], B=\left[\begin{array}{c}
K_{d v} / T_{v} \\
K_{d r} / T_{r} \\
0 \\
w_{n}^{2} K_{d p} \\
0
\end{array}\right], C=\left[\begin{array}{cc}
0 & 0 \\
1 / T_{r} & 0 \\
0 & 0 \\
0 & w_{n}^{2} \\
0 & 0
\end{array}\right],
$$


where $T_{v}$ and $T_{r}$ denote time constants of transfer functions; $\zeta$ and $w_{n}$ denote the damping ratio and the natural frequency under no damping, respectively; $K_{v r}, K_{v p}, K_{d v}, K_{d r}$, and $K_{d p}$ denote given gains.

\section{LEADER-FOLLOWING CONSENSUS PROTOCOL}

Consider a collection of USVs consisting of $\mathrm{N}$ following USVs and one leader USV, where each USV is considered as a node in graph G. According to (2), the follower USV's dynamics is described by:

$$
\dot{x}(t)=A x_{i}(t)+B u_{i}(t)+C w(t), i=1,2, \ldots, N
$$

Without loss of generality, the leading vehicle is labelled $i=0$ and its dynamics is expressed by :

$$
\dot{x}_{0}(t)=A x_{0}(t)
$$

It is assumed that the leader does not receive any information from the followers, i.e. it has no neighbours. A diagonal matrix $M=\operatorname{diag}\left\{m_{1}, m_{2}, \ldots, m_{N}\right\} \in \mathrm{R}^{N \times N}$ is referred to be the leader adjacency matrix with $m_{i} \geq 0$ for any $i$. If the leader is a neighbour of node $v_{i}, m_{i}>0$, otherwise, $m_{i}=0$. In this paper, we assume that node 0 is globally reachable in the union graph $U_{r=1}^{q} G_{r}$. Then the Laplace matrix of the graph $G$ can be represented as $H=L+M \in \mathbb{R}^{N \times N}$.

As mentioned above, the communication channel is subject to packet dropout, channel fading and external disturbances, which may yield intermittent failures in wireless communication between USVs. At a deeper level, this challenge translates into the change of network topology of MUSV systems. Due to the randomness of communication failure, it is assumed that the topology of MUSV systems is steered by a continuous-time Markov process, i.e., $G(\theta(t)) \in\left\{G_{1}, G_{2} \ldots, G_{q}\right\}$ where $\theta(t)$ is a continuous-time Markov process with values in a finite set $\Theta=\{1,2, \ldots, q\}$. The transition probabilities are defined as follows :

$$
\operatorname{prob}\{\theta(t+\Delta t)=s \mid \theta(t)=r\}= \begin{cases}\pi_{r s} \Delta t+o(\Delta t), & r \neq s \\ 1+\pi_{r r} \Delta t+o(\Delta t), & r=s\end{cases}
$$

where $\Delta t>0, o(\Delta t) \rightarrow 0$ as $\Delta t \rightarrow 0$ and $\pi_{r s}$ is the transition rate from mode $r$ to mode $s$, which satisfies $\pi_{s s}=-\sum_{s=1, s \neq r}^{q} \pi_{r s}$ for $r \in \Theta$. Then it is easily known that $H(\theta(t)) \in\left\{H_{1}, H_{2} \ldots, H_{q}\right\}$.

In this paper, we present the following sampled-data leaderfollowing consensus controller for each USV system :

$$
\begin{aligned}
u_{i}(t)=K( & \theta(k h)) \sum_{j=1, j \neq i}^{N} w_{i j}(\theta(k h))\left[x_{i}(k h)-x_{j}(k h)\right] \\
& +K(\theta(k h)) m_{i}(\theta(k h))\left[x_{i}(k h)-x_{0}(k h)\right],
\end{aligned}
$$

where: $K \theta(k h)$ is a consensus controller gain matrix to be designed later, $k h \leq t<(k+1) h, k \in \mathbb{N}, h$ is the sampling period.

Defining the error variables $e_{i}(t)=x_{i}(t)-x_{0}(t)$ for $i=1,2, \ldots, N$ and substituting (5) into (3), one can obtain:

$$
\begin{array}{r}
\dot{e}_{i}(t)=A e_{i}(t)+B K(\theta(k h)) \sum_{j=1, j \neq i}^{N} w_{i j}(\theta(k h))\left[e_{i}(k h)-e_{j}(k h)\right] \\
+B K(\theta(k h)) m_{i}(\theta(k h)) e_{i}(k h)+C w(t)
\end{array}
$$

Let's define an "artificial delay" as $d(t)=t-k h, t \in[k h, k h+h)$. Apparently, it is clear that $d(t)$ is piecewise-linear and $0 \leq d(t)<h$. Then, defining $e(t)=\left[e_{1}(t), e_{2}(t), \ldots, e_{N}(t)\right]^{T}$ and along with (6), the closed-loop error system can be written as follows:

$$
\begin{aligned}
& \dot{e}(t)=\left(I_{N} \otimes A\right) e(t)+\left(H_{r} \otimes B K_{r}\right) e(t-d(t)) \\
& +\left(I_{N} \otimes C\right) w(t), \quad k h \leq t<(k+1) h, \quad k \in \mathbb{N}
\end{aligned}
$$

where the matrix $H_{r}=H(\theta(k h))$ and $K_{r}=K(\theta(k h))$ for each fixed $\theta(k h)=r$.

Next, we introduce the following definition for the consensus problem investigated in this paper.

Definition 1: The closed-loop error system (7) with Markov protocol $\theta(t)$ is said to be robustly stochastic leader-following consensus if the closed-loop error system (7) with $w(k)=0$ is robustly stochastically stable, i.e. for all finite initial condition $\phi\left(t_{0}\right)$ and any given initial $\theta\left(t_{0}\right)$, there exists a finite number $\mu\left(t_{0}, \phi\left(t_{0}\right), \theta\left(t_{0}\right)\right)>0$ such that:

$$
\lim _{t \rightarrow \infty} E\left\{\int_{0}^{t}\|e(t)\|^{2}\right\}<\mu\left(t_{0}, \varphi\left(t_{0}\right), \theta\left(t_{0}\right)\right)
$$

holds, where $E$ is the statistical expectation operator.

Our objective here is to design $K_{r}$ such that the MUSV system can reach robustly stochastically leader-following consensus with the $H_{\infty}$ performance constraint satisfied. In other words, we aim to design a controller such that the closed-loop error system (7) satisfies the following requirements:

Q1. The closed-loop error system (7) with $w(k)=0$ is robustly stochastically stable.

Q2. The effect of $w(t)$ on $e(t)$ is attenuated at a desired level in the $H_{\infty}$ sense. In particular, it is required that:

$$
\|e(t)\|_{2}<\gamma\|w(t)\|_{2}
$$

for all non-zero $w(t) \in L_{2}\left[t_{0}, \infty\right)$ at zero initial condition, where $\gamma>0$.

\section{CONSENSUS ANALYSIS AND CONTROLLER DESIGN}

In this section, we will derive a sufficient condition on the stability of system (7). Then based on the stability criterion in Theorem 1, we are in position to give a sufficient condition on the existence of sampled-data consensus controller gains for MUSV systems.

Lemma 1 [14]. For any constant matrix $R>0$, scalar $\tau>0$ and vector function $\dot{e}:[-h, 0]$ such that the following integration is well defined, then: 
$-h \int_{-h}^{0} \dot{e}^{T}(t+\alpha) \operatorname{Re}(t+\alpha) d \alpha \leq-[e(t)-e(t-h)]^{T} R[e(t)-e(t-h)]$

Theorem 1. For given positive scalars $h$ and $\gamma$, the closedloop error system (7) is robustly stochastically stable with an $H_{\infty}$ norm bound $\gamma$, if there exist symmetric positive definite matrices $P_{r}, Q, R, W$ of appropriate dimensions, such that (8) holds for every feasible value $r \in \Theta$

$$
\left[\begin{array}{cc}
\Pi_{11}^{r} & \Pi_{12}^{r} \\
* & \Pi_{22}
\end{array}\right]<0,
$$

where

$$
\begin{aligned}
& \Pi_{22}=\operatorname{diag}\{-R,-W\}, F_{r}=\left[I_{N} \otimes A-H_{r} \otimes\left(B K_{r}\right) \quad 0 I_{N} \otimes C\right] \text {, } \\
& \mathrm{E}_{r}=\left(I_{N} \otimes A\right)^{T} P_{r} \\
& +P_{r}\left(I_{N} \otimes A\right)+\sum_{s=1}^{q} \pi_{r s} P_{s}+Q-R-\frac{\pi^{2}}{4} W+\gamma^{-1} I, \\
& \Pi_{11}^{r}=\left[\begin{array}{cccc}
\mathrm{E}_{r} & -P_{r}\left(H_{r} \otimes B K_{r}\right)+\frac{\pi^{2}}{4} W & R & P_{r}\left(I_{N} \otimes C\right) \\
* & -\frac{\pi^{2}}{4} W & 0 & 0 \\
* & * & -Q-R & 0 \\
* & * & * & -\gamma I
\end{array}\right]
\end{aligned}
$$

Proof: Choose a Lyapunov-Krasovskii functional as:

$$
V(t, e, \theta)=V_{1}(t, e, \theta)+V_{2}(t, e, \theta)
$$

where:

$V_{1}(t, e, \theta)=e^{T}(t) P_{\theta(t)} e(t)+\int_{t-h}^{t} e^{T}(s) Q e(s) d s+h \int_{-h}^{0} \int_{i+\alpha}^{t} e^{T}(s) R \dot{e}(s) d s d \alpha$,

$V_{2}(t, e, \theta)=-\frac{\pi^{2}}{4} \int_{k h}^{t}[e(s)-e(k h)]^{T} W[e(s)-e(k h)] d s+h^{2} \int_{k h}^{t} \dot{e}^{T}(s) W \dot{e}(s) d s$,

$P_{\theta(t)}, Q, R, W>0$

Then the weak infinitesimal operator of the stochastic process $\theta(t)$ acting on $V(t, e, \theta)$ at the point $\theta(t)=r$, is given by:

$$
L[V(t, e, r)]
$$$$
=\dot{e}^{T}(t) P_{r} e(t)+e^{T}(t) P_{r} \dot{e}(t)+\sum_{s=1}^{q} \pi_{r s} e^{T}(t) P_{s} e(t)+e^{T}(t) Q e(t)
$$$$
-e^{T}(t-h) Q e(t-h)+h^{2} \dot{e}(t) \operatorname{Re}(t)-h \int_{-h}^{0} \dot{e}^{T}(t+\alpha) \operatorname{Re}(t+\alpha) d \alpha
$$$$
-\frac{\pi^{2}}{4}[e(t)-e(k h)]^{T} W[e(t)-e(k h)]+h^{2} \dot{e}^{T}(t) W \dot{e}(t)
$$$$
<\dot{e}^{T}(t) P_{r} e(t)+e^{T}(t) P_{r} e(t)+e^{T}(t)\left(\sum_{s=1}^{q} \pi_{r s} P_{s}+Q\right) e(t)
$$$$
-e^{T}(t-h) Q e(t-h)+h^{2} \dot{e}^{T}(t)(R+W) \dot{e}(t)
$$$$
-[e(t)-e(t-h)]^{T} R[e(t)-e(t-h)]
$$$$
-\frac{\pi^{2}}{4}[e(t)-e(t-d(t))]^{T} W[e(t)-e(t-d(t))] .
$$

Combining (10) and the system (7) together, one has:

$$
\begin{gathered}
L[V(t, e, \theta)]+\gamma^{-1} e^{T}(t) e(t)-\gamma w^{T}(t)< \\
\xi^{T}(t)\left(\Pi^{r}-\Pi_{11}^{r}{ }_{12} \Pi^{-1}{ }_{22} \Pi_{12}^{r}\right) \xi(t)
\end{gathered}
$$

where:

$$
\xi(t)=\left[\begin{array}{llll}
e^{T}(t) & e^{T}(t-d(t)) & e^{T}(t-h) & w^{T}(t)
\end{array}\right] .
$$

By using Schur's complement, one can see that if (8) is satisfied, $\Pi_{11}^{r}-\Pi_{12}^{r} \Pi_{22}^{-1}\left(\Pi_{12}^{r}\right)^{T}<0$ is also satisfied. Then for $t \in[k h, k h+h)$, if (8) is satisfied, one has:

$$
L[V(t, e, \theta)]+\gamma^{-1} e^{T}(t) e(t)-\gamma w^{T}(t) w(t)<0,
$$

If $w(k)=0$, then from (11), we can have that:

$$
L[V(t, e, \theta)]<-\beta_{1}\|\xi(t)\|^{2}<0,
$$

where $\beta_{1}=\min \left(\lambda_{\min }\left(-\Pi_{11}^{r}+\Pi_{12}^{r} \Pi^{-1}{ }_{22}\left(\Pi_{12}^{r}\right)^{T}\right)\right)>0$, $\left.\lambda_{\min }\left(-\Pi_{11}^{r}+\Pi_{12}^{r} \Pi^{-1}{ }_{22}\left(\Pi_{12}^{r}\right)^{T}\right)\right)$ is the minimum eigenvalue of $-\Pi_{11}^{r}+\Pi_{12}^{r} \Pi_{22}^{-1}\left(\Pi_{12}^{r}\right)^{T}$. By Dynkin's formula, we have:

$$
\begin{aligned}
& E\{V(t, e, \theta)\}-V(0, e(0), \theta(0)) \\
& =E\left\{\int_{0}^{t} L[V(s, e, \theta(s))]\right\} d s \\
& <-\beta_{1} \int_{0}^{t} E\left\{\|\xi(s)\|^{2}\right\} d s \\
& <-\beta_{1} \int_{0}^{t} E\left\{\|e(s)\|^{2}\right\} d s
\end{aligned}
$$

On the other hand, we can show that:

$$
E\{V(t, e, r)\}>\beta_{2} E\left\{\|e(t)\|^{2}\right\},
$$

where $\left.\beta_{2}=\min \left(\lambda_{\min }\left(P_{r}\right)\right)>0, \lambda_{\min }\left(P_{r}\right)\right)$ is the minimum eigenvalue of $P_{r}$. The above two inequalities imply that:

$$
E\left\{\|e(t)\|^{2}\right\}<-\kappa_{1} \int_{0}^{t} E\left\{\|e(t)\|^{2}\right\} d s+\kappa_{2} V\left(t_{0}, e\left(t_{0}\right), \theta\left(t_{0}\right)\right)
$$

where: $\kappa_{1}=\beta_{1} \beta_{2}^{-1}>0, \kappa_{1}=\beta^{-1}>0$.

Then, by Gronwall-Bellman lemma [15], we can obtain:

$$
E\left\{\|e(t)\|^{2}\right\}<\kappa_{2} e^{-\kappa_{1} t} V\left(t_{0}, e\left(t_{0}\right), \theta\left(t_{0}\right)\right),
$$

which after integration equals to:

$$
E\left\{\int_{0}^{t}\|e(s)\|^{2} d s\right\}<\kappa_{1}^{-1} \kappa_{2}\left(1-e^{-\kappa_{1} t}\right) V\left(t_{0}, e\left(t_{0}\right), \theta\left(t_{0}\right)\right) .
$$

By taking limit of (13) as $t \rightarrow \infty$, the following is yielded:

$$
\lim _{t \rightarrow \infty} E\left\{\int_{0}^{t}\|e(s)\|^{2} d s\right\}<\kappa_{1}^{-1} \kappa_{2} V\left(t_{0}, e\left(t_{0}\right), \theta\left(t_{0}\right)\right)
$$

Note that $V\left(t_{0}, e\left(t_{0}\right), \theta\left(t_{0}\right)\right)>0$, then by Definition 1 , we know that system (7) is robustly stochastically stable.

If $w(k) \neq 0$, from (11) and (12), one has $\|e(t)\|_{2}<\gamma\|w(t)\|_{2}$. Then, if (8) is satisfied, the system (7) is robustly stochastically stable with an $H_{\infty}$ norm bound $\gamma$. This completes the proof. 
Remark 1. Theorem 1 gives sufficient conditions on the stability of MUSV systems with Markov switching topologies and wave-induce disturbance. The condition of the criterion is formulated in the form of linear matrix inequalities (LMI). Due to the introduction of interior-point methods, the criterion in Theorem 1 can be solved numerically and efficiently. However, instead, if the condition of the criterion is formulated in the other form, such as an algebraic Riccati-type equation, it is very difficult to find solutions except for a few very special cases.

Remark 2: It is the first time when wave-induced disturbance and Markov switching topologies are considered and addressed in the same framework of MUSV systems. The problem investigated here is not only more challenging and complex but is also essentially different in the several aspects from existing ones such as [7].

1) Different problems: Reference [7] discussed consensus problem of a general nonlinear multi-agent systems with Markov switching topologies and communication delay. However, in this paper, we investigate the consensus problem in MUSV systems with Markov switching topologies and wave-induced disturbance.

2) Different objects: Reference [7] discussed the stability of multi-agent system solely, while in this paper, we aim to investigate the stability and robustness of MUSV systems together.

3) Different mathematic techniques: In the LyapunovKrasovskii functional candidate, reference [7] only considered the general item $V_{1}(t, e, \theta)$, while in this paper, the discontinuous Lyapunov-functional $V_{2}(t, e, \theta)$ is also considered, which may lead to a better result and lower computation complexity than the one using only the general item $[16,17]$.

Based on Theorem 1, we can now design the consensus controller (5). Pre and post-multiplying (8) with $\operatorname{diag}\left\{P_{r}^{-1}\right.$, $\left.P_{r}^{-1}, P_{r}^{-1}, I, R^{-1}, W^{-1}\right\}$ and its transposes, and define $P_{r}=I_{N} \otimes \Psi_{r}$, $X_{r}^{r}=\Psi_{r}^{-1}, P_{r}^{-1} Q P_{r}^{-1}=\widetilde{Q}_{r}, P_{r}^{-1} R P_{r}^{-1}=R_{r}, P_{r}^{-1} W P_{r}^{-1}=\widetilde{W}_{r}$ and $Y_{r}=K_{r} X_{r}^{r}$, we are now in position to present the following protocol design criterion.

Theorem 2. For given positive scalar $\gamma$, the leader-following consensus for MUSV systems (3) and (4) can be robustly stochastically achieved in $H_{\infty}$ sense, if there exist real matrices, $\widetilde{Q}_{r}>0, Y_{r}, \widetilde{R}_{r}>0$ and $\widetilde{W}_{r}>0$ with appropriate dimensions such that:

$$
\left[\begin{array}{cccc}
\bar{\Pi}_{11}^{r} & \bar{\Pi}_{12}^{r} & \bar{\Pi}_{13}^{r} & \bar{\Pi}_{14}^{r} \\
* & \bar{\Pi}_{22}^{r} & 0 & 0 \\
* & * & \bar{\Pi}_{33}^{r} & 0 \\
* & * & * & -\gamma I
\end{array}\right]<0
$$

where:

$$
\begin{aligned}
& \bar{\Pi}_{12}^{r}=\left[h \bar{F}_{r}^{T}, h \bar{F}_{r}^{T}\right], \quad r=1,2, \ldots, q, \\
& \bar{F}_{r}=\left[I_{N} \otimes\left(A X_{r}\right)-H_{r} \otimes\left(B Y_{r}\right) 0 I_{N} \otimes C\right], \bar{\Pi}_{14}^{r}=I_{N} \otimes X_{r},
\end{aligned}
$$

$$
\begin{gathered}
\bar{\Pi}_{22}^{r}=\operatorname{diag}\left\{-P_{r}^{-1} \widetilde{R}_{r}^{-1} P_{r}^{-1},-P_{r}^{-1} \tilde{W}_{r}^{-1} P_{r}^{-1}\right\} \\
\bar{\Pi}_{13}^{r}=\left[\sqrt{\pi_{r 1}} X_{r} \ldots \sqrt{\pi_{r r-1}} X_{r} \sqrt{\pi_{r+1}} X_{r} \ldots \sqrt{\pi_{r q}} X_{r}\right], \\
\bar{\Pi}_{33}^{r}=\operatorname{diag}\left\{-X_{1}, \ldots,-X_{r-1},-X_{r+1}, \ldots-X_{q}\right\}, \\
\overline{\mathrm{E}}_{r}=\left(I_{N} \otimes A X_{r}\right)^{T}+I_{N} \otimes A X_{r}+\pi_{r r} X_{r}+\widetilde{Q}_{r}-\widetilde{R}_{r}-\frac{\pi^{2}}{4} \tilde{W}_{r} \\
\bar{\Pi}_{11}^{r}=\left[\begin{array}{cccc}
\overline{\mathrm{E}}_{r} & -H_{r} \otimes\left(B Y_{r}\right)+\frac{\pi^{2}}{4} \tilde{W}_{r} & \widetilde{R}_{r} & I_{N} \otimes C \\
* & -\frac{\pi^{2}}{4} \tilde{W}_{r} & 0 & 0 \\
* & * & -\widetilde{Q}_{r}-\widetilde{R}_{r} & 0 \\
* & * & * & -\gamma I
\end{array}\right] .
\end{gathered}
$$

Moreover, the consensus controller gains are given by: $K_{r}=Y_{r} X_{r}^{-1}$.

Remark 3. The conditions in Theorem 2 include the parameters of network topology, the Markov transmission probabilities and the consensus controller gain. Thus, it is very convenient to employ Theorem 2 for the design of controller gain. Moreover, to handle the nonlinear terms $P_{r}^{-1} \widetilde{R}_{r}^{-1} P_{r}^{-1}$, $P_{r}^{-1} \widetilde{W}^{-1} P_{r}^{-1}$ in (14), one can use the inequalities $-P_{r}^{-1} \widetilde{R}_{r}^{r} P_{r}^{-1} \leq-2\left(I_{N} \otimes X\right)+\widetilde{R}$ and $-P_{r}^{-1} \widetilde{W}_{r}^{-1} P_{r}^{-1} \leq-2\left(I_{N} \otimes X\right)+\widetilde{W}$. Then the nonlinear matrix inequalities can be transformed into strict LMIs. Therefore, for given network topology $G_{r}$ and transition probabilities $\pi_{r s}$, one can obtain the consensus controller gain $K_{r}$ based on the above formulated operation.

\section{SIMULATION}

For the system matrices $A, B$, and $C$ in (2), choose the parameters as follows (see also [18]):

$$
\begin{gathered}
U=7.8(\mathrm{~m} / \mathrm{s}), T_{v}=8 / U, T_{r}=8 / U, \\
K_{d v}=0.01 U, K_{d r}=-0.6027 U, K_{d p}=-0.0014 U^{2}, \\
K_{v r}=-0.46(\mathrm{~m} / \mathrm{s}), K_{v p}=-0.21 U, w_{n}=0.63(\mathrm{rad} / \mathrm{s}) \\
\xi=0.86+0.0038 U .
\end{gathered}
$$

The external wave-induced disturbance is given by :

$$
\left\{\begin{array}{l}
w_{\psi}=2 \cos (2 t) \\
w_{\phi}=-\cos (t)
\end{array}\right.
$$

All the possible information transmission relationships among USVs are given as a group of directed graphs which are shown in Fig. 1. Apparently, the following vehicles can achieve the information from the leading vehicle in Graph $G_{1}$ but lose connection with the leading vehicle in Graph $G_{2}$. Then we know that the leader node is globally reachable in the union graph of $G_{1}$ and $G_{2}$. The corresponding Laplace matrix can be written as:

$$
H_{1}=\left[\begin{array}{cccc}
2 & 0 & 0 & -1 \\
-1 & 2 & 0 & 0 \\
0 & -1 & 1 & 0 \\
0 & 0 & -1 & 1
\end{array}\right], H_{2}=\left[\begin{array}{cccc}
1 & 0 & 0 & -1 \\
-1 & 1 & 0 & 0 \\
0 & -1 & 1 & 0 \\
0 & 0 & -1 & 1
\end{array}\right] \text {. }
$$




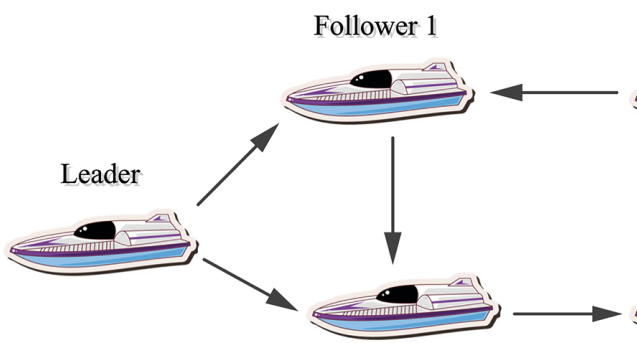

Follower 2

(1) Graph $G_{1}$

Follower 1

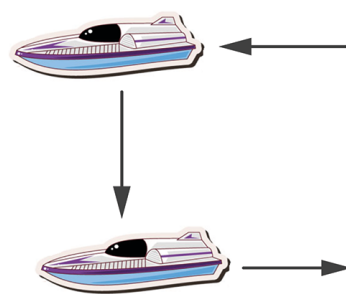

Follower 2

(2) Graph $G_{2}$

Fig. 1. All the possible directed graphs

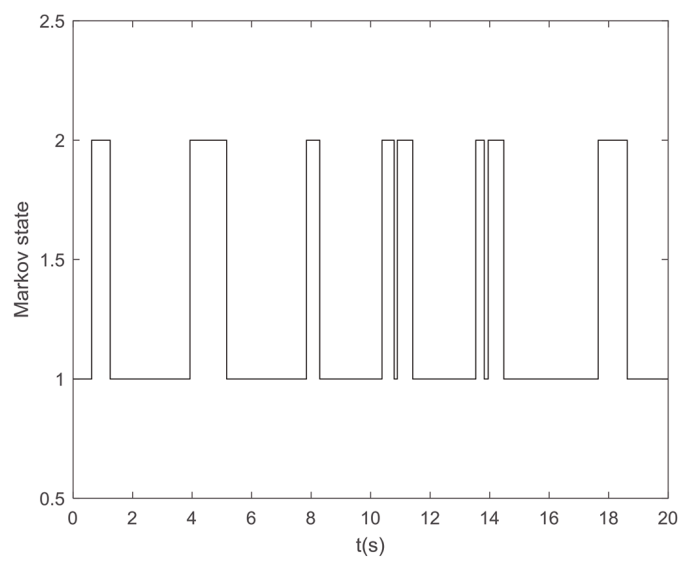

Fig. 2. Markov states of the switching network topologies

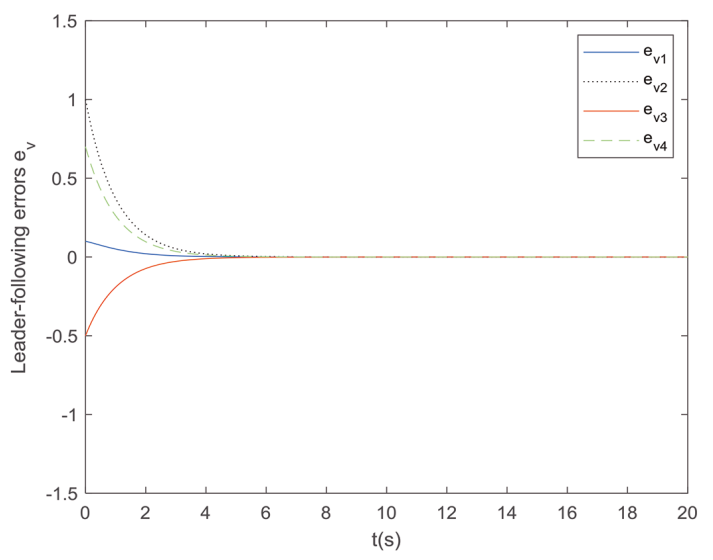

Fig. 3. The responses of the error $v_{i}(t)-v_{0}(t)$
Without loss of generality, it is assumed that all the weights are equal to 1 . The network topologies of the MUSV systems are switched by a Markov chain with the transition matrix

$$
\left[\pi_{s r}\right]=\left[\begin{array}{cc}
-0.5 & 0.5 \\
1 & -1
\end{array}\right]
$$

By solving LMIs in Theorem 2 with $h=0.3$ and $r=2$, the controller gains are obtained as follows:

$$
\begin{gathered}
K_{1}=\left[\begin{array}{lllll}
-0.0576 & 0.1589 & 0.1867 & -0.0001 & 0
\end{array}\right], \\
K_{2}=\left[\begin{array}{lllll}
-0.0638 & 0.1783 & 0.2058 & 0.0003 & 0.0001
\end{array}\right] .
\end{gathered}
$$

By using Matlab software, the Markov states of the switching network topologies are obtained as shown in Fig. 2, where 0 and 1 in the y-axis denote the topology taking values in $G_{1}$ and $G_{2}$, respectively. The responses of MUSV error systems are depicted in Figs. 3, 4, 5, 6 and 7. Due to the derivative relations between the yaw velocity error $r_{i}(t)-r_{0}(t)$ and the heading angle error $\psi_{i}(t)-\psi_{0}(t)$, the changes of the two errors go in the opposite directions; so as the roll velocity error $p_{i}(t)-p_{0}(t)$ and the roll angle error $\phi_{i}(t)-\phi_{0}(t)$. From Figs. 3, 4, 5, 6 and 7, we can clearly see that all the above mentioned errors of MUSV asymptotically converges to zero within 6 seconds, though the followers often

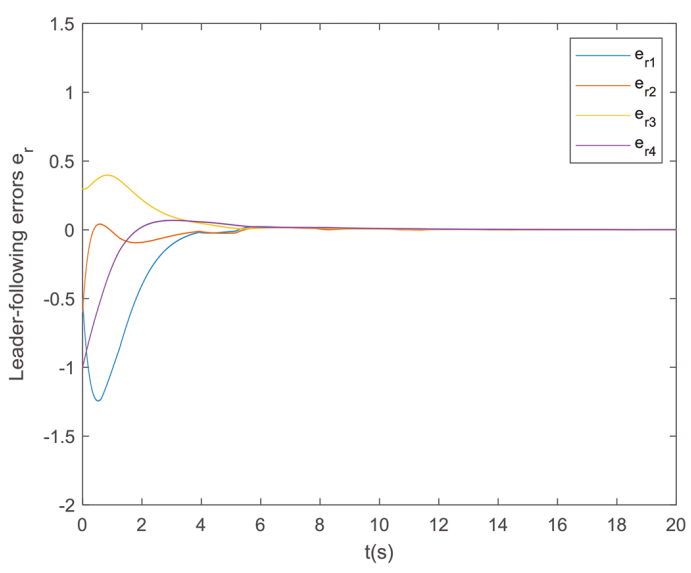

Fig. 4. The responses of the error $r_{i}(t)-r_{0}(t)$

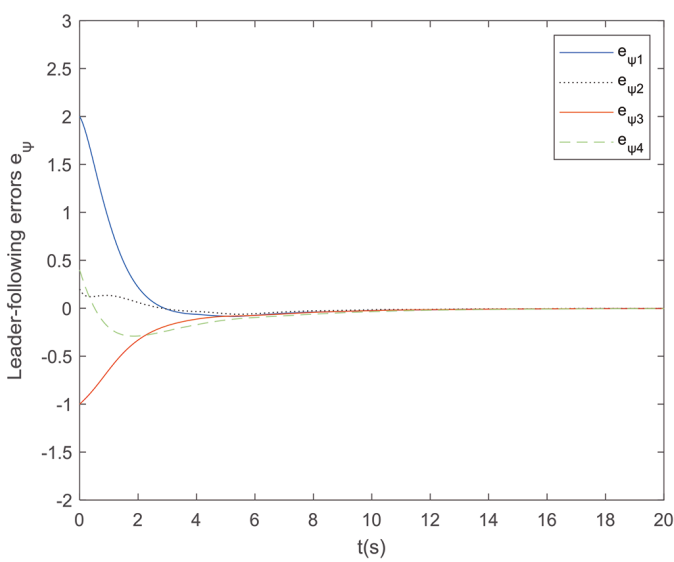

Fig. 5. The responses of the error $\psi_{i}(t)-\psi_{0}(t)$ 


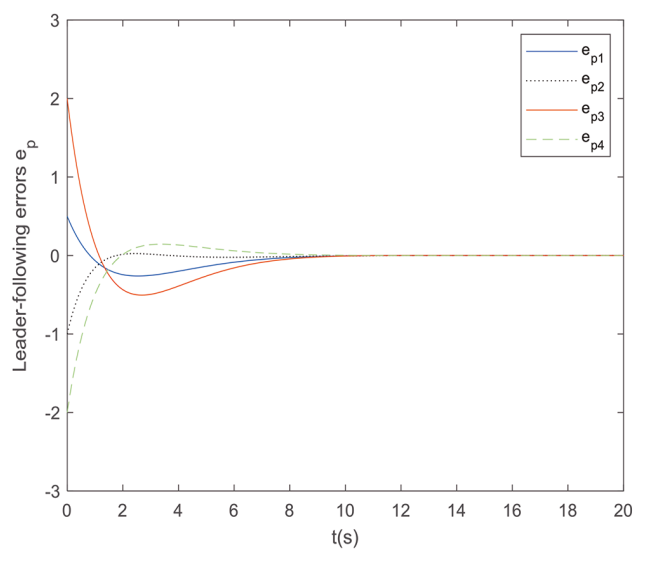

Fig. 6. The responses of the error $p_{i}(t)-p_{0}(t)$

lost connection with the leader vehicle and were affected by the wave-induce disturbance. Therefore we can conclude that the following USV's states indeed reach consensus with the leader USV's state, which illustrates the effectiveness of the proposed design method.

\section{CONCLUSION AND FUTURE WORK}

This paper studied sampled-data leader-following consensus of MUSV systems with Markov network switching topologies and wave-induced disturbance. A new sampled-data consensus protocol which can guarantee that the USV formation system achieve consensus robustly, has been proposed. It is The first time the leader-following consensus problem in MUSV systems has been discussed confirming the results of stability and robustness of multi-agent systems to the actual engineering situation in the marine environment with randomly switching topologies and wave-induced disturbance. A numerical example is given to verify the methods and the simulation results show that the control method can make the following vehicles keep consensus with their leading vehicle. Future research includes the modelling of MUSV system subject to wind-induced disturbance and the designing of consensus protocol constraint by the actuator saturation such as the maximum angle of steering gear.

\section{ACKNOWLEDGEMENTS}

This work is supported by the National Natural Science Foundation of China under Grant No. 61703072 and 61673084, and the Fundamental Research Fund for the Central Universities under Grant No. 3132017128.

\section{REFERENCES}

1. Luca C., Fabio M., Domenico P., and Mario T.: Leader-follower formation control of nonholonomic mobile robots with input constraints. Automatica, 2008, 44(5): pp. 1343-1349.

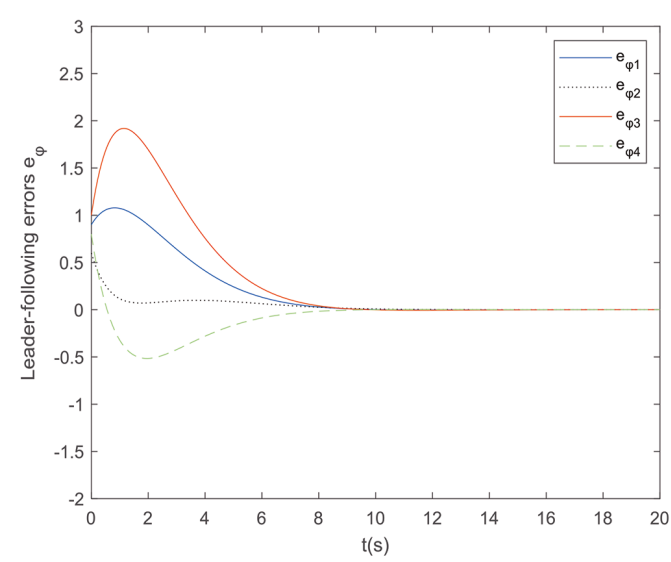

Fig. 7. The responses of the error $\phi_{i}(t)-\phi_{0}(t)$

2. Xing L. T., Wen C. Y., Guo F. H., Liu Z. T., and Su H. Y.: Event-based consensus for linear multi-agent systems without continuous communication. IEEE Transactions on Cybernetics, 2017, 47(8): pp. 2132-2142.

3. Yu X, Liu L, Feng G. Leader-following consensus of multiple unmanned aerial vehicles with input constraints and local coordinate frames. 2016 IEEE International Conference on Advanced Intelligent Mechatronics, 2016, pp. 51061-1066.

4. Jia Y. N., Wang L.: Leader-follower flocking of multiple robotic fish. IEEE/ASME Transactions on Mechatronics, 2015, 20(3): pp. 1372-1383.

5. Liu Z. Q., Wang Y. L., Wang T. B.: Incremental predictive control-based output consensus of networked unmanned surface vehicle formation systems. Information Science, 2018, 457-458: pp. 166-181.

6. Yi J. W., Wang Y. W., Xiao J. W.: Consensus in Markovian jump second-order multi-agent systems with random communication delay. IET Control Theory and Application, 2014, 8(16): pp. 1666-1675.

7. Ding L., Guo G.: Sampled-data leader-following consensus for nonlinear multi-agent systems with Markovian switching topologies and communication delay. Journal of the Franklin Institute, 2015, 352: pp. 369-383.

8. Kaviarasan B., Sakthivel Chao Wang C., Alzahrani F.: Resilient control design for consensus of nonlinear multi-agent systems with switching topology and randomly varying communication delays. Neurocomputing, 2018, 311: pp. 155-163.

9. Li C. J., Liu G. P.: Consensus for heterogeneous networked multiagent systems with switching topology and time-varying delays. Journal of the Franklin Institute, 2018, 355: pp.4198-4217.

10. Dai J. T., Guo G.: Event-triggered leader-following consensus for multi-agent systems with semi-Markov switching topologies. Information Sciences, 2018, 459: pp. 290-301. 
11. Van der Klugt P. G. M.: Rudder Roll Stabilization. Ph. D. dissertation, Faculty Elect. Eng., Math. Comput. Sci., Delft Univ. Technol., Delft, The Netherlands, 1987.

12. Van Amerongen J., Van der Klugt P. G. M., and Van Nauta Lemke H. R.: Rudder roll stabilization for ships. Automatica, 1990, 26(4): pp. 679-690.

13. Wang Y. -L., Han Q. -L.: Network-based heading control and rudder oscillation reduction for unmanned surface vehicles. IEEE Transactions Control Systems Technology, 2017, 25(5): pp. 1609-1620.

14. Han Q.-L.: Absolute stability of time-delay systems with sector-bounded nonlinearity. Automatica, 2005, 41(12): pp. 2171-2176.

15. Bellman R., Stability theory of differential equations, McGraw-Hill, New York, 1953.

16. Peng C., Zhang J., Han Q. -L.: Consensus of multi-agent systems with nonlinear dynamics using an integrated sampleddata-based event-triggered communication scheme. IEEE Transactions on Systems, Man, And Cybernetics systems, 2018, (online).

17. Zhenman G., Yong H., Min, W.: New constructing method of Lyapunov-Krasovskii functionals for stability of time-varying delay systems. IECON $2017-43^{\text {rd }}$ Annual Conference of the IEEE Industrial Electronics Society, 2017, pp. 5639-5643.

18. Fossen T. I.: Guidance and Control of Ocean Vehicles. Hoboken, NJ, USA: Wiley, 1994.

\section{CONTACT WITH THE AUTHORS}

\author{
Liyuan Wang \\ e-mail:wangliyuandmu@163.com \\ Dalian Minzu University \\ Liaohe West Road \\ Jinzhou New District, 116600 Dalian \\ China \\ Wei Yue \\ e-mail:yuewei811010@163.com \\ Dalian Maritime University \\ Ganjingzi Street, 116026 Dalian \\ China \\ Rubo Zhang \\ e-mail:zhangrubo@dlnu.edu.cn \\ Dalian Minzu University \\ Liaohe West Road \\ Jinzhou New District, 116600 Dalian \\ China
}

